\title{
Three Dimensional Simulations of Fire Plume Dynamics
}

\author{
H.R. BAUM, K.B. McGRATTAN and R.G. REHM \\ National Institute of Standards and Technology \\ Gaithersburg, Maryland 20899 USA
}

\begin{abstract}
The velocity and temperature fields of fire plumes are studied theoretically and computationally using the authors' large eddy simulation techniques. A version of the Navier-Stokes equations specialized to the study of fire dynamics is used as the basis for the analysis. No empirical turbulence modeling parameters are introduced into the computations. These simplifications permit high resolution solutions to the three dimensional time-dependent Navier-Stokes to be directly compared with experimental correlations of the time-averaged properties of an isolated vertical plume. Comparisons are made by averaging the time-dependent numerical results rather than the governing equations. Computed mean velocities and temperatures are shown to be in close agreement with McCaffrey's correlations. The methodology is then applied to the study of a large pool fire in an aircraft hangar with a complicated roof geometry. A simple cell masking scheme permits the inclusion of the building geometry into the calculation without sacrificing computational efficiency. The plume structure in the hangar is compared with the isolated plume properties.
\end{abstract}

KEY WORDS: Buoyant Convection, Computational Fluid Dynamics, Fires, Fire-Induced Flows, Plumes.

\section{INTRODUCTION}

The fire plume is the single most important object of study in fire science. It provides the radiant energy source that generates the gasified fuel from the condensed phase needed to sustain the fire. It also serves as the heat pump which both entrains air into the active combustion zone and then circulates the combustion products through the surrounding enclosure. It is the latter role that is of interest here. Although the central role of the fire plume has been recognized for some time, there is still much uncertainty about the plume structure. Indeed, Zukoski [1] gives a recent summary of the state of knowledge of mass entrainment into isolated fire plumes. 
There is clearly no consensus on any simple formula or graphical correlation for this most basic of plume quantities. Much of this uncertainty arises from the experimental and conceptual difficulty associated with determining the outer plume boundary. McCaffrey [7] developed centerline mean velocity and temperature correlations that are consistent with a large body of experimental data [8].

When the plume interacts with the enclosure geometry, the concept of an isolated plume becomes more difficult to sustain. Hiroomi et al. [2] have demonstrated systematic changes in observed plume structure as a result of confinement in tall, narrow enclosures. Bilger [3] has advocated the use of field models, in particular those based on $k-\epsilon$ models of turbulence, to study issues of this type. Although there has been much progress using this approach to study fire induced flows in enclosures, most of the effort has concentrated on regions outside the plume itself. An excellent example of this is the analysis of the the experiments of Steckler et al. [5, 6] by Kerrison et al. [4]. The disadvantage of using such an experiment as a test of fire-induced flow models is that it is difficult to determine how much the $k-\epsilon$ model, the choice of boundary condition, the representation of the enclosure geometry, or the experimental data contribute to those discrepancies between computed and measured results.

The present work uses the large eddy simulation techniques developed by the authors to study the fire plume both as an isolated entity and in a realistic enclosure environment. The purpose is twofold - to contribute to the understanding of fire plume dynamics and to demonstrate the power of the large eddy simulation technique. The absence of boundaries, the fact that a reasonable (albeit imperfect) data base exists for an isolated plume, and the fact that an explicit turbulence model is not used in the present approach, makes this an attractive problem to consider. The choice of the enclosure fire scenario mentioned below demonstrates that this methodology can be applied to problems of practical interest. In the next section, the three dimensional transient Navier-Stokes equations specialized to fire scenarios are presented. The numerical techniques used to solve them are briefly outlined. Following this, high resolution solutions for a purely buoyant fire plume are presented and compared with McCaffrey's centerline correlations. Finally, the methodology is applied to the analysis of a pool fire in an aircraft hangar. The spatial resolution is high enough to have the plume resolved nearly as well in this complex geometry as in the isolated plume case. The two plumes are compared, and the effect of the enclosure is shown. A concluding section discusses the future prospects for this approach to fire dynamics.

\section{MATHEMATICAL MODEL}

We consider a thermally expandable ideal gas driven by a prescribed heat source. The equations of motion governing the fluid flow are written in a form suitable for low Mach number applications [9]. Sometimes, this form of the equations is referred to as "weakly compressible". The most important feature of these equations is that in the energy conservation equation the spatially and temporally varying pressure is replaced by an average pressure $p_{0}$ which depends only on time. This is done to filter out acoustic waves. The efficiency of the numerical solution of the equations is dramatically increased by this approximation.

In the equations to follow, all symbols have their usual fluid dynamical meaning: $\rho$ is the 
density, $\mathbf{u}$ the velocity vector, $\boldsymbol{\omega}$ the vorticity, $p$ the pressure, $\mathbf{g}$ the gravity vector, $c_{p}$ the constant-pressure specific heat, $T$ the temperature, $k$ the thermal conductivity, $t$ the time, $\dot{q}$ the prescribed volumetric heat release, $\mathcal{R}$ the gas constant equal to the difference of the specific heats $\mathcal{R}=c_{p}-c_{v}$, and $\boldsymbol{\sigma}$ the standard stress tensor for compressible fluids.

$\frac{\partial \rho}{\partial t}+\nabla \cdot \rho \mathbf{u}=0$

$\rho\left(\frac{\partial \mathbf{u}}{\partial t}+\frac{1}{2} \nabla|\mathbf{u}|^{2}-\mathbf{u} \times \boldsymbol{\omega}\right)+\nabla p-\rho \mathbf{g}=\nabla \cdot \boldsymbol{\sigma}$

$\rho c_{p}\left(\frac{\partial T}{\partial t}+\mathbf{u} \cdot \nabla T\right)-\frac{d p_{0}}{d t}=\dot{q}+\nabla \cdot k \nabla T$

$p_{0}(t)=\rho \mathcal{R} T$

The divergence of the flow $\nabla \cdot \mathbf{u}$ is a very important quantity in the analysis to follow, and it is readily found by combining Eqs. (1) and (3) and using the equation of state (4)

$p_{0} \nabla \cdot \mathbf{u}+\frac{1}{\gamma} \frac{d p_{0}}{d t}=\frac{\gamma-1}{\gamma}(\dot{q}+\nabla \cdot k \nabla T)$

where $\gamma=c_{p} / c_{v}$. Integrating Eq. (5) over the entire domain $\Omega$ yields a consistency condition for the background pressure $p_{0}(t)$

$p_{0} \int_{\partial \Omega} \mathbf{u} \cdot d \mathbf{S}+\frac{V}{\gamma} \frac{d p_{0}}{d t}=\frac{\gamma-1}{\gamma}\left(\int_{\Omega} \dot{q} d V+\int_{\partial \Omega} k \nabla T \cdot d \mathbf{S}\right)$

where $V$ is the volume of the enclosure. The background pressure can be expressed in terms of a background temperature $T_{0}(t)$ and density $\rho_{0}(t)$

$p_{0}=\mathcal{R} \rho_{0} T_{0}$

These spatially averaged quantities play the same role that ambient conditions do in the Boussinesq approximation. Perturbations to each are represented by the relations

$T=T_{0}(t)(1+\tilde{T}) \quad ; \quad \rho=\rho_{0}(t)(1+\tilde{\rho})$

The perturbation values are thus simply related

$(1+\tilde{T})(1+\tilde{\rho})=1$

Defining $T_{0}$ and $\rho_{0}$ through the adiabatic process

$\frac{\rho_{0}}{\rho_{\infty}}=\left(\frac{p_{0}}{p_{\infty}}\right)^{1 / \gamma}$ 
allows the energy equation to be expressed in terms of the perturbation temperature $\tilde{T}$ and the divergence

$$
\frac{\partial \tilde{T}}{\partial t}+\mathbf{u} \cdot \nabla \tilde{T}=(1+\tilde{T})\left[\nabla \cdot \mathbf{u}+\frac{1}{\gamma p_{0}} \frac{d p_{0}}{d t}\right]
$$

The background pressure is found from Eq. (6).

Next, we simplify the momentum equation. The pressure is composed of three components, the background $p_{0}(t)$, the hydrostatic, and a perturbation to the hydrostatic $\tilde{p}$

$p(\mathbf{r}, t)=p_{0}(t)-\rho_{0}(t) g z+\tilde{p}(\mathbf{r}, t)$

where $z$ is the vertical spatial component. We subtract off the hydrostatic pressure gradient from the momentum equation (2), and then divide by the density to obtain

$$
\frac{\partial \mathbf{u}}{\partial t}+\frac{1}{2} \nabla|\mathbf{u}|^{2}-\mathbf{u} \times \boldsymbol{\omega}+\frac{1}{\rho} \nabla \tilde{p}-\frac{\rho-\rho_{0}}{\rho} \mathbf{g}=\frac{1}{\rho} \nabla \cdot \boldsymbol{\sigma}
$$

To simplify this equation further, the density in the pressure term is assumed ambient, and then the term $|\mathbf{u}|^{2} / 2$ is combined with the perturbation pressure $\tilde{p} / \rho_{0}$ and written as atal pressure, $\mathcal{H}$. This approximation assumes that the buoyancy generated vorticity dominates the baroclinic contribution caused by the non-alignment of perturbation pressure and density gradients. This is not a good approximation at the small scales where the actual combustion heat release takes place. However, at the resolvable scales this is nothing more than the assumption that these are buoyancy dominated flows. If the coefficient of viscosity is assumed constant, the momentum equation can be written

$$
\frac{\partial \mathbf{u}}{\partial t}-\mathbf{u} \times \boldsymbol{\omega}+\nabla \mathcal{H}+\tilde{T} \mathbf{g}=\nu\left(\frac{4}{3} \nabla(\nabla \cdot \mathbf{u})-\nabla \times \boldsymbol{\omega}\right)
$$

For most applications, the effective kinematic viscosity coefficient $\nu$ represents dissipation on length scales below the resolution limits of the calculation. The effective Reynolds number is high enough to permit direct simulation of convective motion over a spatial range of two orders of magnitude for a three-dimensional calculation.

The fire is represented by introducing a large number of Lagrangian elements which release heat as they are convected about by the thermally induced motion. Since the fluid motion determines where the heat is actually released, and the heat release determines the motion, the large scale features of the coupling between the fire and the smoke transport are retained. It should be noted, however, that the heat release rate is not predicted, but is an input parameter in the computer programs implementing this model. The smoke is simulated by tracking the convected elements after the fuel burnout is completed. A specified percentage of the fuel consumed is assumed to be converted to smoke particulate. Thus, a knowledge of the spatial distribution of the Lagrangian elements is equivalent to a specification of the smoke particulate density at any instant of time. This "thermal element" model, which represents the combustion heat release as a large number of point sources convected by the resolvable flow field, is in fact a simple combustion model in its own right. It is consistent with more detailed combustion 


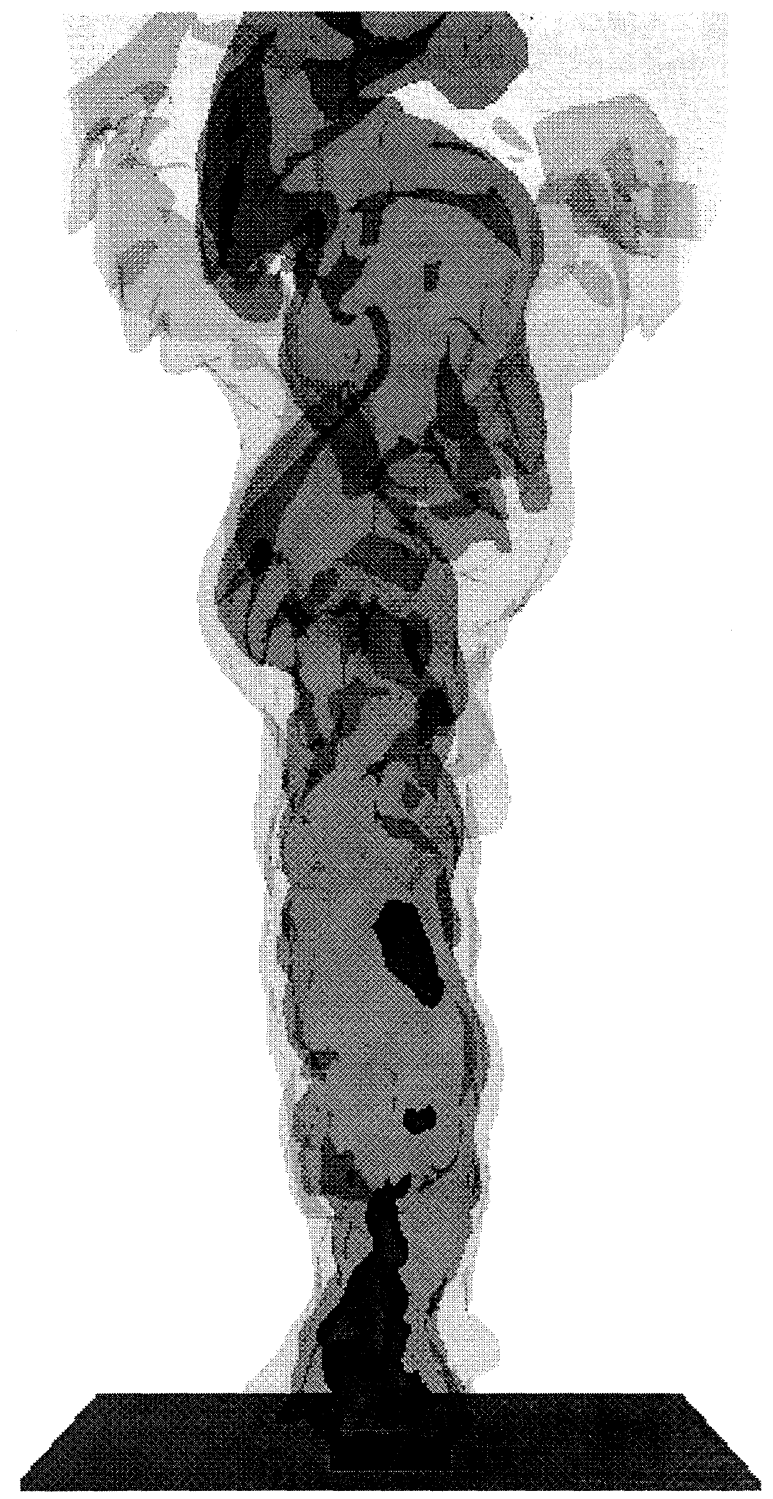

FIGURE 1: Instantaneous snapshot of a pool fire calculation performed on a $96 \times 96 \times$ 96 grid. The contours correspond roughly to the boundaries of the continuous flame, intermittent region and plume. The square burner is length $D^{*}$ on each side, with the height of the domain rising about $12 D^{*}$. 
theories currently in use, and it permits the use of experimental data from fire experiments in a way that does not violate the consequences of those theories. A more detailed discussion of this model can be found in Ref. [12].

In summary, the equations which are solved numerically are the energy equation (11), the momentum equation (14), and a Poisson equation for the total pressure, obtained by taking the divergence of Eq. (14). This linear elliptic equation is solved with the use of an FFT-based fast direct solver. The background pressure, temperature and density are found from Eqs. (6), (7) and (10). Each of the conservation equations emphasize the importance of the divergence and vorticity fields, as well as the close relationship between the thermally expandable fluid equations [9] and the Boussinesq equations for which the authors have developed highly efficient solution procedures $[10,11]$. These are applied directly to the equations presented here with minor modifications and no loss in performance. The only changes from earlier methodology are a return to a uniform rectangular grid with blocks of cells masked to simulate internal boundaries; and the use of a second order Runge-Kutta scheme to advance the velocity and temperature fields in time. The speed and accuracy of this technique enable calculations on current generation workstations which involve over a million computational cells, yielding the spatial range of two orders of magnitude for a three-dimensional calculation.

\section{ISOLATED FIRE PLUME}

We first consider an isolated fire plume generated by a pool fire. The minimum length scale that must be resolved here is the plume structure scale $D^{*}=\left(Q / \rho_{0} c_{p} T_{0} \sqrt{ } g\right)^{2 / 5}$. Since $D^{*}$ is roughly comparable to the plume diameter near the base, the plume dynamics can only be accurately simulated if the resolution limit is about $0.1 D^{*}$. Indeed, for an isolated plume, this is the only length scale that must be considered. The physical diameter of the pool enters into any calculation as part of the domain geometry. However, $D^{*}$, which involves the heat release rate $Q$ directly, is the fundamental parameter. This can be seen both from the dimensionless form of McCaffrey's plume correlations [8] and by considering the dimensionless form of the NavierStokes equations for this problem. In fact, if the equations discussed above are made nondimensional with $D^{*}$ as length scale, $\sqrt{ }\left(g D^{*}\right)$ as velocity scale, $\sqrt{ }\left(D^{*} / g\right)$ as time scale, and $T_{0}$ as temperature; all the physical constants disappear from the inviscid terms in the equations. Only Reynolds and Prandtl numbers appear in the viscous stress and heat conduction terms. In all computations, the Reynolds number is chosen to be high enough so that the inviscid terms dominate the large scale structure. The need to accomplish this and also allow the viscous terms to provide dissipation at the resolution limit is the ultimate reason that high spatial and temporal resolution are required.

Figure 1 shows an instantaneous snapshot of three temperature contours obtained from a $96 \times$ $96 \times 96$ cell simulation. The contours correspond to the boundaries of the continuous flame, intermittent flame, and plume zones as defined in Ref. [8]. The Reynolds number was 12,000 for this simulation, based on the height of the computational domain. Note that Fig. 1 is an instantaneous snapshot of the fire, and that time averages of the output of this kind of simulation must be produced in order to make quantitative comparison with most experimental data. Indeed, it is the fact that the results of the simulation can be averaged in a routine way while the equations of fluid mechanics cannot is the basis of the whole approach presented 

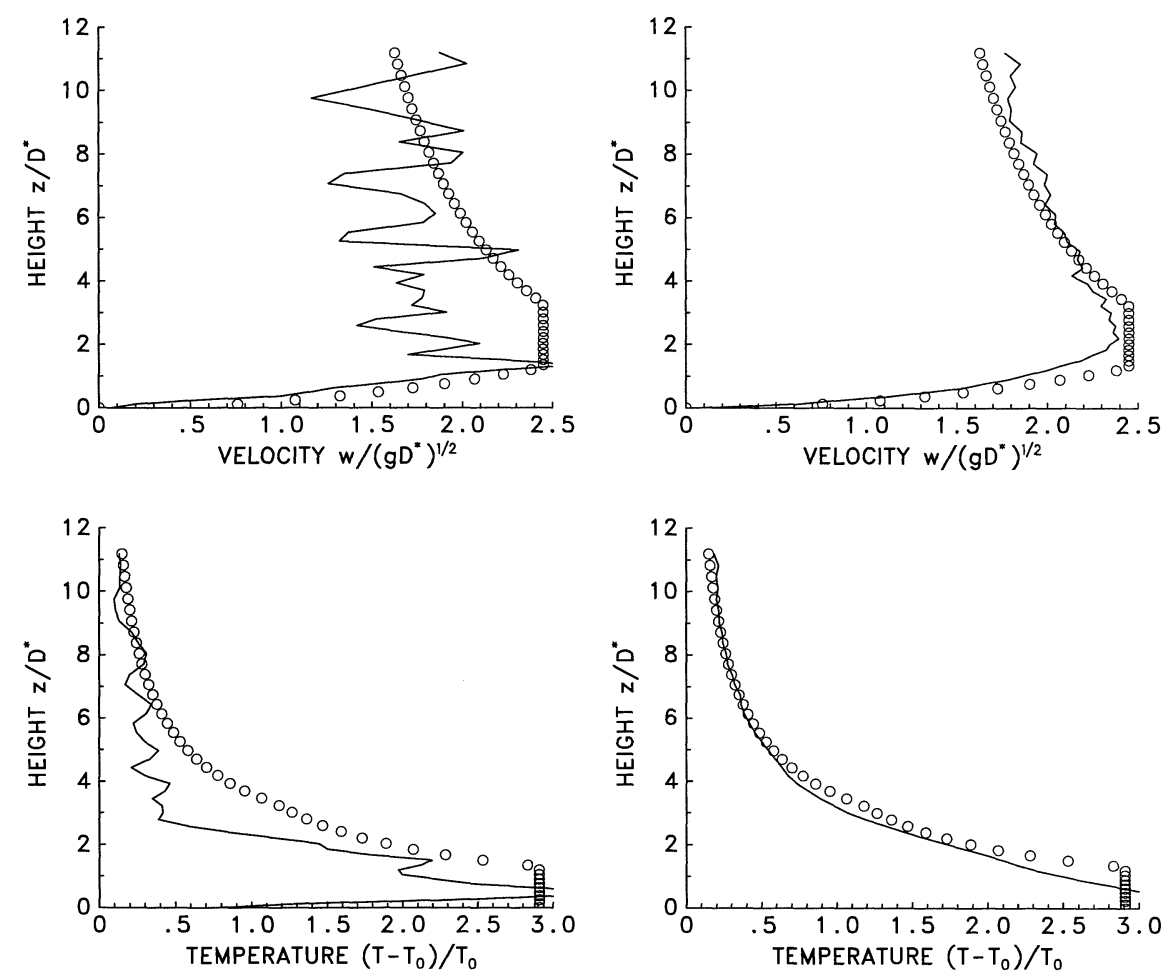

FIGURE 2: Instantaneous (left) and time-averaged (right) centerline velocity and temperature profiles for the pool fire simulation shown in Fig. 1.

here.

On the left of Fig. 2 are the instantaneous vertical centerline velocity and temperature profiles superimposed on the steady-state correlation of McCaffrey (circles). The oscillations are primarily due to the large toroidal vortices generated at regular intervals at the base of the fire, which then rise asymmetrically. Note that the flow is not even remotely axially symmetric, and the centerline is defined only by the geometry of the pool at the base of the plume. The right side of Fig. 2 shows the corresponding time averaged quantities (solid lines) and McCaffrey's centerline correlations. The time averaged flow is symmetric and in excellent agreement with the correlations. The major deviations are at the bottom of the plume where the thermal elements are turned on instantaneously without any preheat as they leave the pool surface. Otherwise, the boundaries of the computational domain are open. At these open boundaries, the perturbation pressure is assumed zero. This is a reasonable approximation at the side boundaries, but less so at the top. Various strategies have been explored to properly set the conditions at the top, but in most instances, there is usually a solid ceiling or a hood drawing the combustion products up at some specified flow rate. Fig. 3 shows the corresponding radial dependence of the vertical velocity and temperature at heights of $z / D^{*}=2.8$ (intermittent region) and 

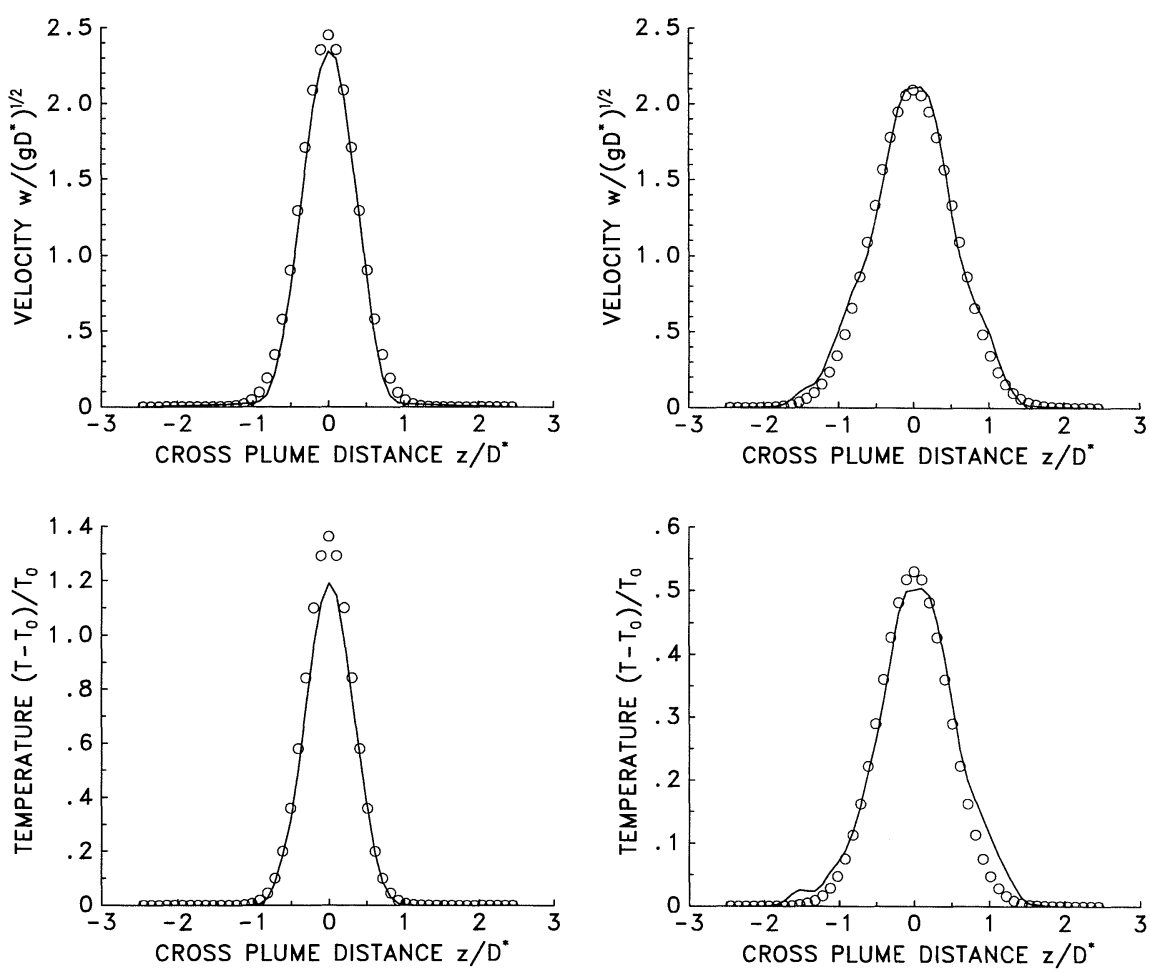

FIGURE 3: Radial profiles of vertical velocity and temperature at vertical locations $z / D^{*}=2.8$ (intermittent region) and $z / D^{*}=5.3$ (plume region) for the pool fire simulation shown in Fig. 1.

$z / D^{*}=5.3$ (plume region). The results of the simulation are compared with Gaussian profiles that incorporate the half-widths obtained from McCaffrey's correlations [8].

\section{AIRCRAFT HANGAR FIRE}

Next, we consider a simulation of one of a series of fire tests performed in a $22 \mathrm{~m}$ high aircraft hangar at the U.S. Naval Air Station in Keflavik, Iceland [13]. The hangar has a floor approximately $75 \mathrm{~m}$ by $45 \mathrm{~m}$ in extent, and a barrel vault ceiling that is divided into five $15 \mathrm{~m}$ sections by draft curtains that all extend down to 16 meters above the floor. The three center sections are represented in a simulation that consists of a $3 \mathrm{~m}$ square JP-5 jet fuel fire (15 MW) located in the geometric center of the hangar. Figure 4 displays an instantaneous picture of the fire and the hanger geometry. The computations were performed on a $180 \times 180 \times 90$ cell grid (roughly $25 \mathrm{~cm}$ resolution); perhaps the largest CFD-based computation of a problem in fire research. They required $3.2 \times 10^{-6}$ seconds per time step per grid cell on a Cray-90 supercomputer. Forty-five seconds of steady burning following a five second ramp-up were simulated. 


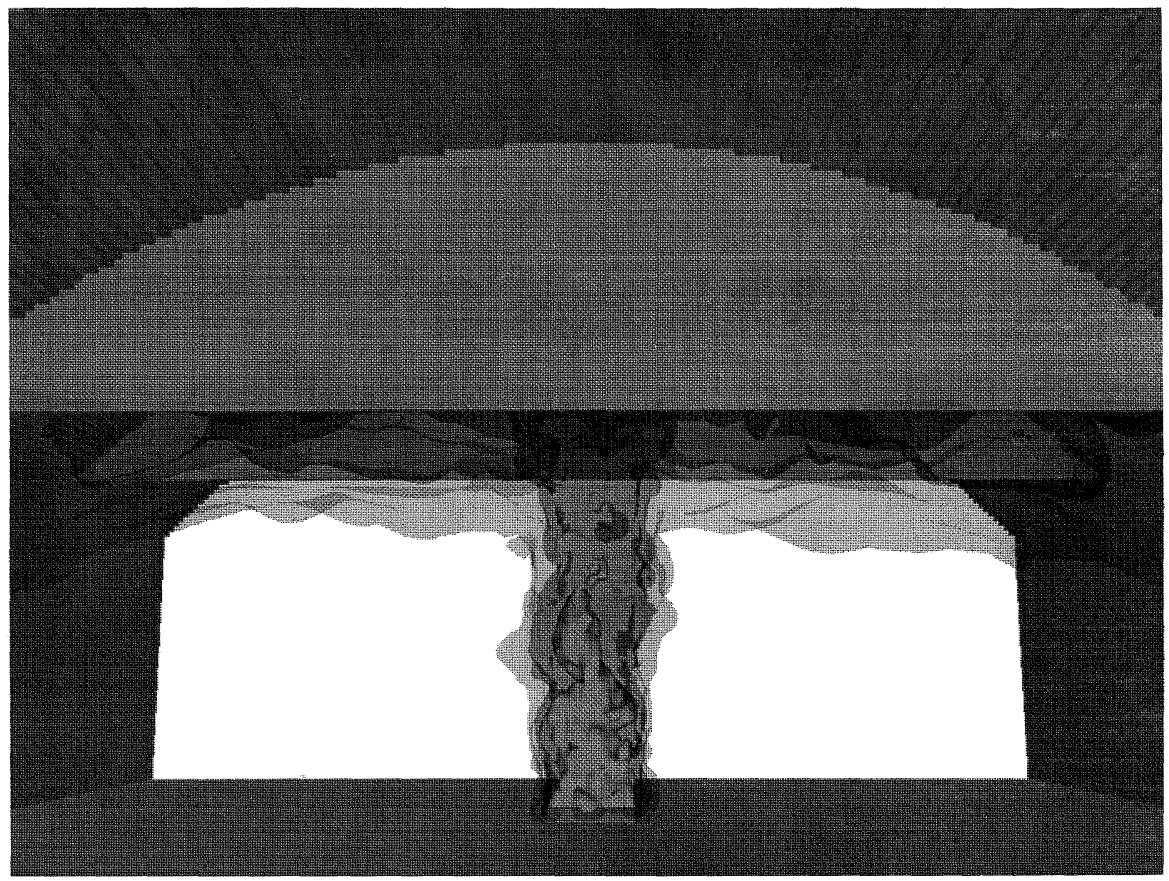

FIGURE 4: Instantaneous snapshot of a fire plume simulation in a $22 \mathrm{~m}$ high aircraft hangar at the U.S. Naval Air Station in Keflavik, Iceland. The width of the building is $\mathbf{4 5}$ $\mathrm{m}$, and $45 \mathrm{~m}$ of its length is included in the calculation. The actual length of the building is about $75 \mathrm{~m}$.

The Reynolds number based on the height of the hangar is 14,000 .

The high resolution calculations were undertaken to ensure that the fire plume in the hangar was resolved to about the same spatial resolution employed in the isolated plume case. Since $D^{*}$ is more than an order of magnitude smaller than the hangar width, the computational requirements for this problem were quite high. In fact, there are not many CFD computations in any applied field where this dynamic range of length scales has been studied in a fully transient three dimensional simulation. One side benefit of the high resolution requirement is that curved surfaces such as the hangar roof can be readily simulated with little loss of accuracy and no loss of computational efficiency. The grid is sufficiently dense so that no point on the curved boundary is more than one half percent of the enclosure height from the nearest computational boundary. Note that much larger enclosures can be simulated so long as the fires of interest are correspondingly larger, and that no small scale geometry need be accounted for. The task of simulating a relatively small fire in a large enclosure is much more difficult. It is usually assumed that the enclosure geometry has little effect once the ratio of $D^{*}$ to a suitable geometric scale is small enough. It is now becoming possible to find out to what extent this assumption is valid. 
Figure 5 shows the time-averaged centerline plume temperatures and vertical velocities in the hangar environment. The isolated plume correlations are also shown for comparison. The time averages were taken for a ten second period about 40 seconds into the simulation. At this time the well mixed upper layer extends to roughly $16 \mathrm{~m}$ above the hangar floor, and spillover has just begun. The maximum temperature at a point $0.15 \mathrm{~m}$ below the ceiling directly above the fire was measured at roughly $170^{\circ} \mathrm{C}$. The corresponding time-averaged calculated temperature from Fig. 5 was $155^{\circ} \mathrm{C}$. We believe this average is equivalent to the measured quantity but there is not enough detail in [13] to be certain. In fact any further comparisons between the calculations and experiments are not possible due to the lack of data.
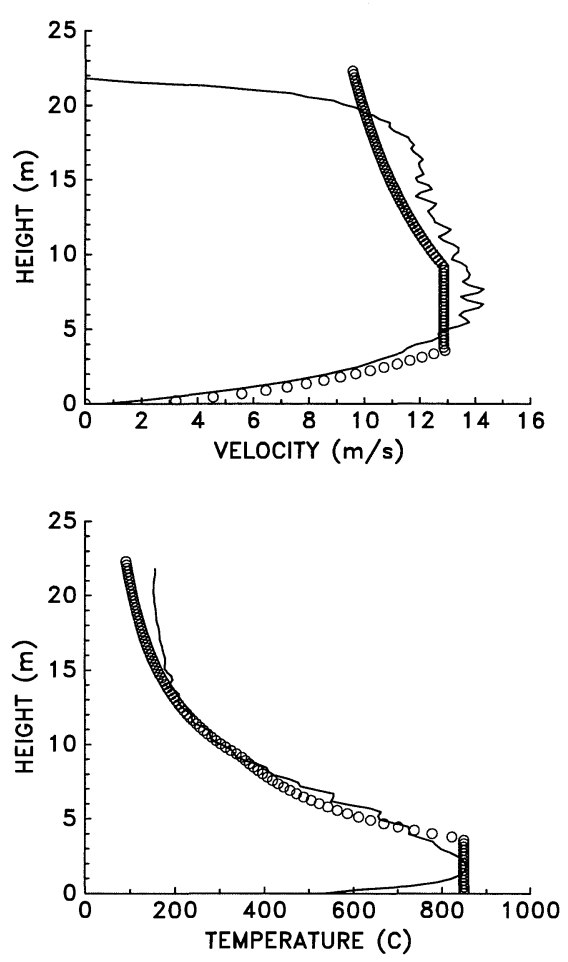

FIGURE 5: Time-averaged centerline vertical velocity and temperature for the hanger simulation shown in Fig. 4.
Clearly, the plume calculated for the hangar scenario is very close to the classical isolated plume up to the point where the stable hot layer begins to interact with the plume. Considering that the simulation was performed for a complex geometry and the correlation is for an isolated plume, the agreement is remarkably close. The plume was assumed to lose thirty-five percent of the chemically released energy through radiation that is lost to the system. These are exactly the assumptions made for the isolated plume study and are implicit in the correlations presented in Ref. [8]. The hangar is thus sufficiently large compared with the fire for the interactions reported in [2] to be unimportant. The placement of the fire in the center of a large space with the outer doors closed undoubtably contributed to the limited influence of enclosure geometry on plume dynamics. Clearly, the presence of draft curtains played little role in the plume dynamics. Their principal effect was to confine the formation of the upper layer at this stage in the fire development.

\section{CONCLUSION}

The computations shown above demonstrate that the large scale fire dynamics and smoke movement generated by realistic fire scenarios can be accurately calculated directly from the Navier-

Stokes equations. The dynamic range of the spatial and temporal resolution achieved is comparable to those used in fundamental studies of the structure of homogeneous turbulence, and greater than that currently employed in predictions of the global atmospheric circulation [14]. At present, the main limitations on the utility of the present approach for problems of practical interest have more to do with the incorporation of other physical processes into the large eddy simulations than with further improvements to the description of turbulent mixing. The most 
important of these within the context of large scale transport phenomena is thermal radiation. Efforts to incorporate radiative transport in a manner consistent with the large eddy simulations described above are currently underway.

\section{REFERENCES}

[1] Zukoski, E.E., "Mass Flux in Fire Plumes", in Fire Safety Science- Proceedings of the Fourth International Symposium, International Association for Fire Safety Science, pp. 137-148, 1994.

[2] Hiroomi, S., Sugawa, O., Kurioka, K., and Takahashi, W., "Plume Behavior in a Confined Tall and Narrow Space-as One of Sub-Models of Plume for an Atrium Fire-", Fire Safety Science- Proceedings of the Fourth International Symposium, International Association for Fire Safety Science, pp. 551-562, 1994.

[3] Bilger, R.W., "Computational Field Models in Fire Research and Engineering", Fire Safety Science- Proceedings of the Fourth International Symposium, International Association for Fire Safety Science, pp. 95-110, 1994.

[4] Kerrison, L., Mawhinney, N., Galea, E.R., Hoffman, N., and Patel, M.K., "A Comparison of Two Fire Field Models with Experimental Room Fire Data", Fire Safety Science- Proceedings of the Fourth International Symposium, International Association for Fire Safety Science, pp. 161-172, 1994.

[5] Steckler, K.D., Quintiere, J.G., and Rinkinen, W.J., "Flow Induced by a Fire in a Compartment", Nineteenth Symposium (International) on Combustion, The Combustion Institute, Pittsburgh, pp. 913-920, 1982.

[6] Steckler, K.D., Baum, H.R., and Quintiere, J.Q., "Fire Induced Flows Through Room Openings", Twentieth Symposium (International) on Combustion, The Combustion Institute, Pittsburgh, pp. 1591-1600, 1984.

[7] McCaffrey, B.J., "Purely Buoyant Diffusion Flames -Some Experimental Results", NBSIR 79-1910, National Bureau of Standards, 1979.

[8] Baum, H.R. and McCaffrey, B.J., "Fire Induced Flow Field- Theory and Experiment", Fire Safety Science- Proceedings of the Second International Symposium, Hemisphere, New York, pp. 129-148, 1989.

[9] Rehm, R.G. and Baum, H.R., "The Equations of Motion for Thermally Driven, Buoyant Flows", Journal of Research of the NBS, Vol. 83, pp. 297-308, 1978.

[10] McGrattan, K.B., Rehm, R.G., and Baum, H.R., "Fire-Driven Flows in Enclosures", J. Comp. Phys., Vol. 110, pp. 285-291, 1994.

[11] Baum, H.R., Ezekoye, O.A., McGrattan, K.B. and Rehm, R.G., "Mathematical Modeling and Computer Simulation of Fire Phenomena", Theoretical and Computational Fluid Dynamics, Vol. 6, pp. 125-139, 1994. 
[12] Baum, H.R., McGrattan, K.B. and Rehm, R.G., "Large Eddy Simulations of Smoke Movement in Three Dimensions", Conference Proceedings of the Seventh International Interflam Conference, Interscience Communications, London, pp. 189-198, 1996.

[13] Notarianni, K.A., Davis, W., Lowe, D., and Laramee, S., "Analysis of High Bay Hangar Facilities for Detector Sensitivity and Placement", Conference Proceedings of the Seventh International Interflam Conference, Interscience Communications, London, pp. 487-496, 1996.

[14] Lorentz, E.N., The Essence of Chaos, University of Washington Press, Seattle, 1993. 